\title{
Allelic variation at the VERNALIZATION-A1, VRN-B1, VRN-B3, and PHOTOPERIOD-A1 genes in cultivars of Triticum durum Desf.
}

\section{Muterko, Alexandr}

2016-12

Muterko , A , Kalendar , R \& Salina , E 2016 , ' Allelic variation at the VERNALIZATION-A1, VRN-B1, VRN-B3, and PHOTOPERIOD-A1 genes in cultivars of Triticum durum Desf. ' , Planta , vol. 244 , no. 6 , pp. 1253-1263 . https://doi.org/10.1007/s00425-016-2584-5

http://hdl.handle.net/10138/308719

https://doi.org/10.1007/s00425-016-2584-5

unspecified

acceptedVersion

Downloaded from Helda, University of Helsinki institutional repository.

This is an electronic reprint of the original article.

This reprint may differ from the original in pagination and typographic detail.

Please cite the original version. 


\title{
Allelic variation at the VERNALIZATION-A1, VRN-B1, VRN-B3, and PHOTOPERIOD-A1 genes in cultivars of Triticum durum Desf.
}

\author{
Alexandr Muterko ${ }^{1,2}\left(\right.$ [D $\cdot$ Ruslan Kalendar ${ }^{3,4} \cdot$ Elena Salina $^{1}$
}

Received: 9 June 2016/Accepted: 9 August 2016/Published online: 13 August 2016

(C) Springer-Verlag Berlin Heidelberg 2016

\begin{abstract}
Main conclusion The durum wheat varieties from Ukraine, Russia, and Kazakhstan are characterized by the specific allelic composition of the $V R N$ genes that sharply distinguish them from the Triticum durum varieties from other countries. For numerous varieties, the $V R N$ alleles which previously were not found in tetraploid wheat were identified.

The ability of wheat to adapt to a wide range of environmental conditions is mostly determined by the allelic diversity within genes regulating the vernalization requirement $(V R N)$ and photoperiod response $(P P D)$. In the present study, allelic variation in the VRN1, VRN3, and PPD-Al genes was investigated for 134 varieties of Triticum durum from different eco-geographic areas. It was shown that varieties from Russia and Ukraine have a specific allelic composition at the VRN genes, which in
\end{abstract}

Electronic supplementary material The online version of this article (doi:10.1007/s00425-016-2584-5) contains supplementary material, which is available to authorized users.

Alexandr Muterko

muterko@bionet.nsc.ru; muterko@gmail.com

1 The Federal Research Center Institute of Cytology and Genetics, Lavrentyeva Avenue 10, Novosibirsk 630090, Russian Federation

2 Plant Breeding and Genetics Institute-National Center of Seed and Cultivar Investigation, Ovidiopolskaya Road 3, Odessa 65036, Ukraine

3 RSE "National Center for Biotechnology", Sh. Valikhanov 13/1, Astana 010000, Kazakhstan

4 MTT Plant Genomics Laboratory, Biocentre 3, Institute of Biotechnology, University of Helsinki, Viikinkaari 1, P.O. Box 65, 00014 Helsinki, Finland quantity and quality differed from European and American cultivars. A large number of varieties of $T$. durum from Russia carry the dominant Vrn-Ala.1 allele, previously identified mainly in hexaploid wheat. For some varieties from Eastern Europe and Asia, Vrn-Ali and vrn-Alb.3 recently revealed in wheat were also identified. Polymorphism of the $V R N-B 1$ promoter region, distinguishing all three variants of this sequence (VRN-B1.f, VRN-B1.s, and $V R N-B 1 . m)$, was detected. It was found that the dominant $V r n-B 1 c$ allele is commonly found in varieties of $T$. durum from Russia and Ukraine, but not Europe or USA. Furthermore, many Ukrainian and Russian varieties carry the dominant alleles of the both $V R N-A 1$ and $V R N-B 1$ genes simultaneously, while varieties from Europe and America carry the dominant allele of $V R N-A l$ alone. Finally, a high frequency of the $V r n-B 3 a$ allele, which previously was found only in some accessions of hexaploid wheat, was observed for varieties from Ukraine and Russia. It was revealed that the Ukrainian pool of $T$. durum varieties is currently the largest genetic source of the dominant Vrn$B 3 a$ allele in wheat in the worldwide.

Keywords Cereal - Durum wheat - Genetic variation . Photoperiod response . Vernalization requirement

\section{Introduction}

The adaptability of wheat to a wide range of environment conditions is mainly controlled by genes, determining the vernalization requirement $(V R N)$ and photoperiod sensitivity $(P P D)$. These agronomically valuable traits are important to ensure the flowering of wheat in the most favorable climatic conditions and are widely used in 
modern breeding to obtain high-yielding varieties that are favorable adapted to changes in environmental conditions.

The requirement of fall-planted winter wheat for a prolonged exposure to low temperatures for timely flowering in spring, a process known as vernalization, is an important adaptation protecting sensitive floral meristems from frost damage during the winter. The vernalization requirement of wheat is mainly controlled by four $V R N$ genes: VRNI (a MADS-box transcription factor) (Yan et al. 2003), VRN2 (a zinc-finger CCT domain gene-ZCCT) (Yan et al. 2004b), VRN3 (TaFT, homologous to the Arabidopsis gene FLOWERING LOCUS T) (Yan et al. 2006), and VRN4 (MADS-box transcription factor, duplication of the VRN-Al gene in the short arm of chromosome 5D) (Kippes et al. 2015). During regulation of vernalization response of wheat, the $V R N 1, V R N 2$, and $V R N 3$ genes interact epistatically to form a feedback regulatory loop in which VRN3 is the integrator of the vernalization and photoperiod floral pathways [reviewed by Muterko et al. (2015a)]. Regulation of the vernalization response in this way enables the developmental stages of wheat to progress in accordance with the seasonal changes in climate.

Vernalization insensitive varieties with a spring growth habit, determined by the recessive vrn2 alleles, require deletions or non-functional mutations in all ZCCT genes simultaneously and have not been observed in naturallyoccurring variations in polyploid wheat (Muterko et al. 2015a). However, allelic variation at the $V R N-B 2$ locus is associated with a partially dominant effect in tetraploid wheat (Distelfeld et al. 2009). VRN4 is mapped to chromosome 5 of the D genome, and is not relevant to the analysis of tetraploid wheat with the BBAA genome. Thus, the vernalization requirement of tetraploid wheat is mainly determined by allelic variation at the VRNI and potentially $V R N 3$ genes. There are numerous reports of allelic variation in VRN genes in Triticum aestivum cultivars and wild tetraploid wheat species. However, no significant investigations were found to identify allelic variation at the VRN genes in varieties of Triticum durum.

VRN1 genes have been mapped to the long arms of the chromosomes 5 homeologous group (Yan et al. 2003) and encode MADS-box transcriptional factors, which are involved in the transition of the apical meristem from the vegetative to reproductive stages (Danyluk et al. 2003). The dominant $V R N-A l$ alleles as a rule result in complete elimination of the vernalization requirement and determine a spring growth habit. The presence of either the dominant $V R N-B 1$ or $V R N-D 1$ alleles alone is associated with some residual vernalization requirement and later flowering that indicates a facultative growth habit. Allelic variations at the VRN1 genes are determined by mutations within the promoter region and intron-1. In hexaploid, wheat dominant $V R N-A 1$ alleles carrying InDels and/or SNP mutations within the promoter region are most common. In contrast, the dominant $V r n-A l$ alleles in tetraploid wheat, depending on species, carry mutations in promoter region or large deletion within intron-1 (Muterko et al. 2016). Furthermore, in winter, varieties of hexaploid wheat (T. aestivum) were identified from one to four copies of the $V R N-A l$ gene (Würschum et al. 2015). This copy number variation (CNV) polymorphism was associated with change in vernalization requirement duration and flowering time of wheat (Díaz et al. 2012). The dominant $V R N-B 1$ alleles are mainly caused by the large deletions within the first intron for both hexaploid and tetraploid wheat (Muterko et al. 2016). However, in the latter study, a polymorphism of the $V R N-B 1$ promoter region associated with early flowering of spring wheat accessions was also identified (Muterko et al. 2016).

Authentic natural variation for VRN3 has been found only in B genome (Yan et al. 2006; Chen et al. 2013). The $V R N-B 3$ gene is the wheat ortholog of the Arabidopsis FT (Flowering locus $T$ ) and being a typical florigene induces flowering (Yan et al. 2006). This gene encodes an RAF kinase inhibitor-like protein acting upstream of $V R N 1$ in a feedback regulatory loop. The dominant allele $V r n-B 3 a$ contains a $5.3 \mathrm{~kb}$ insertion of a retrotransposon in the promoter region, $591 \mathrm{bp}$ from the transcription initiation site that results in overexpression of $T a F T$ and early flowering (Yan et al. 2006). The $V r n-B 3 c$ allele is also dominant and differs from $\mathrm{Vrn}-\mathrm{B} 3 \mathrm{a}$ by deletions of 20 and $4 \mathrm{bp}$ in the retrotransposon sequence. Herewith, these deletions do not influence $V R N-B 3$ transcription level and flowering time in accessions with $V r n-B 3 c$ as compared to $V r n-B 3 a$ (Chen et al. 2013). The dominant $V R N-B 3$ alleles are very rare. Furthermore, allelic variation at the $V R N-B 3$ gene was previously studied for only hexaploid varieties of bread wheat (T. aestivum) and has not been investigated for species of tetraploid wheat and particularly for varieties of T. durum until now.

The sensitivity of wheat to photoperiod indicates the dependence of flowering on day length. Photoperiod response in wheat is mainly determined by the homoeologous series of Photoperiod 1 (PPDI) genes. Recessive $P p d 1 b$ alleles (wild type) confer sensitivity to day length, with flowering delayed under short days (SDs, $<10 \mathrm{~h}$ light), while flowering is promoted under long day (LD) photoperiods ( $\sim 16 \mathrm{~h}$ day lengths). Dominant Ppdla mutants confer photoperiod insensitivity, and result in rapid flowering under both SD and LD photoperiods. A photoperiod insensitive (PI) phenotype in wheat is an important agronomic trait, and for this reason, the use of PI alleles becomes widespread in wheat varieties following the "green revolution" and continues to be widely used globally. In wheat, homoeologous PPD1 genes are located on the short arms of the group 2 chromosomes 
(Beales et al. 2007). Ppdla carry mutations within the promoter region, which cause their overexpression independent of photoperiod. Copy number variation $(\mathrm{CNV}$ mutations) also is a cause of PI phenotype (Beales et al. 2007; Díaz et al. 2012), but this has a lesser effect on decreasing photoperiod sensitivity than PPDI promoter region deletions. Furthermore, $\mathrm{CNV}$ mutants have been identified only for $P P D-B 1$ in hexaploid wheat of $T$. aestivum and were not shown for varieties of T. durum. Although numerous mutations within the $P P D-B 1$ promoter region have been identified in wild emmer wheat, their phenotypic effect is unknown (Takenaka and Kawahara 2012). Thus, currently, the photoperiod insensitive $P P D-B 1$ alleles for varieties of $T$. durum are not known.

The photoperiod insensitive Ppd-Ala.l (1085 bp deletion) and Ppd-Ala.4 (648 bp deletion) alleles previously identified in hexaploid wheat species of T. aestivum and Triticum compactum, respectively (Nishida et al. 2013; Muterko et al. 2015b), while such PI alleles as Ppd-Ala.2 (1027 bp deletion) and Ppd-Ala.3 (1117 bp deletion) were found in isogenic wheat lines of $T$. durum (Wilhelm et al. 2009). All these alleles carry deletions of different lengths within the promoter region and are characterized by differing effects on flowering time during growth of plants under a short photoperiod (Wilhelm et al. 2009; Nishida et al. 2013; Muterko et al. 2015b). Furthermore, based on the change in curvature of the DNA fragments of $P P D-A 1$ that is associated with SNPs in the promoter region, the Ppd-Alb can be divided into two haplogroups, AI and AII (Muterko et al. 2015b).

In our recent studies, 21 accessions of $T$. durum from 20 countries of the world, excluding Russia, Ukraine, and Kazakhstan, were analyzed at the VRNI and $P P D-A 1$ genes (Muterko et al. 2015b, 2016). In the present study, we have expanded the subset of cultivars of T. durum and focused on previously uncharacterized gene varieties from Russia, Ukraine, and Kazakhstan to find how allelic diversity at the $V R N$ and PPD-Al genes of these varieties differs from those in varieties from other countries. We conduct investigation of $T$. durum cultivars by the diagnostic DNA markers and also using the early proposed methods of the detection of the recently identified new $V R N-A 1$ and $V R N-B 1$ alleles (Muterko et al. 2016), which until now were not analyzed in a large set of soft and durum wheat. Furthermore, our study also includes the analysis of the $V R N-B 3$ gene, which previously was not investigated in tetraploid wheat species and particularly in cultivars of $T$. durum. Finally, using a specially developed algorithm of hierarchical clustering, the analysis of the obtained allelic combinations of $V R N$ genes will be grouped to find specific compositions of these alleles for different centers of wheat cultivation.

\section{Materials and methods}

\section{Plant material}

A total of $134 T$. durum varieties from 17 countries, with a focus on Ukrainian and Russian varieties, were investigated (Table S1). Germplasm was obtained from the National Centre for Plant Genetic Resources of Ukraine (to request germplasm of varieties No. 1-75 from Table S1, contact: yuriev1908@gmail.com, ncpgru@gmail.com), Siberian Research Institute for Plant Industry and Breeding (by Vjacheslav Piskarev, piskarev@bionet.nsc.ru; varieties No. 76-122 from Table S1), and Kazakhstan-Siberian Program (by Vladimir Shamanin, shamanin@bionet.nsc.ru; varieties No. 123-134 from Table S1).

\section{DNA extraction and PCR amplification}

Total DNA from 4-day-old wheat seedlings was extracted following a modified CTAB method (Doyle and Doyle 1987). PCR reactions consisted of: DNA ( $\sim 40 \mathrm{ng})$, $20 \mathrm{mM}$ Tris- $\mathrm{HCl}(\mathrm{pH} 8.8), 10 \mathrm{mM}\left(\mathrm{NH}_{2}\right)_{2} \mathrm{SO}_{4}, 2.6 \mathrm{mM}$ $\mathrm{MgCl}_{2}, 1 \mathrm{mM} \mathrm{KCl}, 0.1 \%$ Triton X-100, $250 \mu \mathrm{M}$ dNTPs, $1.5 \%$ DMSO, $3 \mathrm{ng} / \mu \mathrm{l}$ each primer, and $0.05 \mathrm{U} / \mu \mathrm{l} \mathrm{Taq}-$ polymerase. PCR was performed using the following program: denaturation at $94{ }^{\circ} \mathrm{C}(2 \mathrm{~min}) ; 30$ cycles of amplification: $94{ }^{\circ} \mathrm{C}(10 \mathrm{~s})$, annealing $(10 \mathrm{~s}), 72{ }^{\circ} \mathrm{C}(50 \mathrm{~s}$ or 2 min for amplification of the $V R N-B 3$ promoter region and $V R N-B 1$ intron-1) per cycle, 3 cycles: annealing (10 s), $72{ }^{\circ} \mathrm{C}(60 \mathrm{~s})$, and a final elongation step of $72{ }^{\circ} \mathrm{C}$ for 3 min. Further details of all primers, including annealing temperatures, are listed in Table 1.

\section{Polyacrylamide and agarose gel electrophoresis}

PCR fragments of the $V R N-A 1$ and $V R N-B I$ first intron and $V R N-B 3$ promoter region were separated on $6.6 \%$ nondenaturing polyacrylamide (PAA) gels (mono/bis-acrylamide ratio 82:1) in $1.38 \times$ TBE buffer (123 $\mathrm{mM}$ ionic strength), at room temperature, under $6 \mathrm{~V} / \mathrm{cm}$. For the separation of the $V R N-A 1, V R N-B 1$, and $P P D-A 1$ promoter region amplicons, the polyacrylamide gel electrophoresis (PAGE) was carried out as described previously (Muterko et al. 2016). Agarose gel electrophoresis was performed using $1.5 \%$ agarose in $1 \times$ TBE buffer. Visualization of PCR fragments in PAA and agarose gels was conducted using ethidium bromide under UV light.

\section{Sequencing of PCR fragments}

PCR fragments were excised from agarose gel and purified on silica spin columns according to the manufacturer's 
protocol. Sequencing was carried out with the use of BigDye Terminator v3.1 sequencing kit with the subsequent analysis on an ABI $3130 \times 1$ Genetic Analyzer. The partial $V R N-A 1, V R N-B 1$, and $V R N-B 3$ sequences reported in this paper were deposited in GenBank under accession numbers: KU738895 (Kharkovskaya 1; vrn-Alb.3), KX139167 (GK Basa; Vrn-Ali), KX139174 (Bashkirskaya 27; VrnAla.1), KX268729 (Zenati 368; Vrn-Alb.1), KX139169KX139170 (Marzaga, Lavina; Vrn-Alb.6), KX139168
(Hordeiforme 18567-6; VRN-B1.m), KX139171 (Elizavetinskaya; Vrn-B3a), KX139173 (Donskaya Elegiya; VrnB1c), and KX139172 [Kargala 28; Vrn-Alc (Langdon)].

\section{Data analyses}

To perform cluster analysis, an algorithm of the controlled sequential hierarchical clustering was developed (Supplemental Material S2). Undirected graphs generated during

Table 1 PCR primers details

\begin{tabular}{|c|c|c|c|c|c|c|}
\hline Primers & Primer sequence $\left(5^{\prime}-3^{\prime}\right)$ & Primer design & $\begin{array}{l}\text { Annealing } \\
\text { temp. }{ }^{\circ} \mathrm{C}\end{array}$ & Amplified region & Allelic variant & $\begin{array}{l}\text { PCR } \\
\text { product size } \\
(\mathrm{bp})\end{array}$ \\
\hline $\begin{array}{l}\text { VRN1AF } \\
\text { VRN1-INT1R }\end{array}$ & $\begin{array}{l}\text { gaaaggaaaaattctgctcg } \\
\text { gcaggaaatcgaaatcgaag }\end{array}$ & Yan et al. (2004a) & 60 & $\begin{array}{l}V R N-A 1 \\
\text { promoter }\end{array}$ & $\begin{array}{l}\text { vrn-Al } \\
\text { Vrn-Ala.1 } \\
\text { Vrn-Ala.2 } \\
\text { Vrn-Ala.3 } \\
\text { Vrn-Alb } \\
\text { Vrn-Ald } \\
\text { Vrn-Ale } \\
\text { Vrn-Alf } \\
\text { Vrn-Ali } \\
\text { vrn-A } A^{m} 1 \\
\text { Vrn-A } A^{m} 1 g \\
\text { Vrn-A } A^{m} 1 a \\
v r n-A^{m} 1 b\end{array}$ & $\begin{array}{r}713 \\
944 \\
924,944 \\
765 \\
691 \\
685 \\
659 \\
658 \\
713 \\
705 \\
681 \\
671 \\
656\end{array}$ \\
\hline $\begin{array}{l}\text { Vrn-A1-intr_F } \\
\text { Vrn-A1-intr_R1 }\end{array}$ & $\begin{array}{l}\text { ccgtcgaaaggatcgctactg } \\
\text { cttgtccecgtgagctacttac }\end{array}$ & Muterko et al. (2016) & 60 & $V R N-A 1$ intron-1 & $v r n-A 1$ & 541 \\
\hline $\mathrm{Ex} 1 / \mathrm{C} / \mathrm{F}$ & gttctccaccgagtcatggt & Fu et al. (2005) & 56 & $V R N-A 1$ intron-1 & $\begin{array}{l}\text { Vrn-Alc } \\
\quad(\text { Langdon })\end{array}$ & 522 \\
\hline $\begin{array}{l}\text { Intr1/A/R3 } \\
\text { Pr1 } \\
\text { Pr2 }\end{array}$ & $\begin{array}{l}\text { aagtaagacaacacgaatgtgaga } \\
\text { taccctgctaccagtgcct } \\
\text { ggccaaccctacaccccaag }\end{array}$ & Shcherban et al. (2012) & 58 & $\begin{array}{l}V R N-B 1 \\
\text { promoter }\end{array}$ & $\begin{array}{l}\text { Vrn-A1c (IL369) } \\
V R N-B 1 . f \\
V R N-B 1 . s \\
V R N-B 1 . m\end{array}$ & $\begin{array}{r}2188 \\
968 \\
958 \\
965\end{array}$ \\
\hline $\begin{array}{l}\mathrm{Ex} 1 / \mathrm{C} / \mathrm{F} \\
\text { Intr1/B/R3 }\end{array}$ & $\begin{array}{l}\text { gttctccaccgagtcatggt } \\
\text { ctcatgccaaaaattgaagatga }\end{array}$ & Fu et al. (2005) & 58 & $V R N-B 1$ intron-1 & $\begin{array}{l}\text { Vrn-B1a } \\
\text { Vrn-B1b } \\
\text { Vrn-B1c }\end{array}$ & $\begin{array}{r}1091 \\
1055 \\
705\end{array}$ \\
\hline $\begin{array}{l}\mathrm{Ex} 1 / \mathrm{C} / \mathrm{F} \\
\text { Intr1/B/R4 }\end{array}$ & $\begin{array}{l}\text { gttctccaccgagtcatggt } \\
\text { caaatgaaaaggaatgagagca }\end{array}$ & Fu et al. (2005) & 60 & $V R N-B 1$ intron-1 & $v r n-B 1$ & 1531 \\
\hline $\begin{array}{l}\text { FT-B-INS-F } \\
\text { VRN4-B-NOINS-R }\end{array}$ & $\begin{array}{l}\text { cataatgccaagccggtgagtac } \\
\text { ctatccctaccggccattag }\end{array}$ & Yan et al. (2006) & 61 & $\begin{array}{l}V R N-B 3 \\
\text { promoter }\end{array}$ & $V r n-B 3 a$ & 1765 \\
\hline $\begin{array}{l}\text { VRN4-B-NOINS- } \\
\text { F2 } \\
\text { VRN4-B-NOINS-R }\end{array}$ & $\begin{array}{l}\text { getgtgtgatcttgctctcc } \\
\text { ctatccetaccggccattag }\end{array}$ & Yan et al. (2006) & 61 & $\begin{array}{l}V R N-B 3 \\
\text { promoter }\end{array}$ & $v r n-B 3$ & 691 \\
\hline $\begin{array}{l}\text { durum_Ag5del_F2 } \\
\text { durum_Ag5del_R2 }\end{array}$ & $\begin{array}{l}\text { cgtcacccatgcactctgtt } \\
\text { ctggctccaagaggaaacac }\end{array}$ & Wilhelm et al. (2009) & 56 & $\begin{array}{l}P P D-A 1 \\
\text { promoter }\end{array}$ & $\begin{array}{l}p p d-A 1 b \\
p p d-A^{m} 1 b\end{array}$ & $\begin{array}{l}452 \\
456\end{array}$ \\
\hline $\begin{array}{l}\text { Ppd-A1proF } \\
\text { durum_Ag5del_R2 }\end{array}$ & $\begin{array}{l}\text { gtgtcgcacggattttgctc } \\
\text { ctggctccaagaggaaacac }\end{array}$ & $\begin{array}{l}\text { Muterko et al. (2015b) } \\
\text { Wilhelm et al. (2009) }\end{array}$ & 56 & $\begin{array}{l}P P D-A 1 \\
\text { promoter }\end{array}$ & $\begin{array}{l}\text { Ppd-Ala.1 } \\
\text { Ppd-Ala.2 } \\
\text { Ppd-Ala.3 } \\
\text { Ppd-Ala.4 }\end{array}$ & $\begin{array}{l}405 \\
463 \\
372 \\
806\end{array}$ \\
\hline
\end{tabular}


cluster analysis were visualized using Dendroscope 2.5 (Huson et al. 2007).

\section{Results}

Three primer pairs were used to detect the allelic variants of the VRN-Al gene (Table 1). Although primer pair VRN1AF and VRN1-INT1R flanks more than 460 bp of the promoter region, exon-1 and part of intron-1 the amplicon length polymorphism until now was found to associate with mutations within promoter region and was not shown for covered transcribed region. This allows us to differentiate more than ten promoter alleles of $V R N-A 1$ based on the difference in amplicon lengths. Furthermore, the informativeness of some DNA markers was significantly increased by carrying out of PAA electrophoresis under special conditions, allowing the allocation of no less than seven additional allelic variants of $V R N-A 1$, and avoiding the sequencing and errors associated with it (Muterko et al. 2016). The presence or absence of large deletions within $V R N-A 1$ intron-1 was defined using separate PCRs with primer pairs Ex1/C/F, Intr1/A/R3 and Vrn-A1-intr_F, Vrn-A1-intr_R1. The 522 bp fragments, amplified with primers Ex1/C/F and Intr1/A/R3, indicate the Vrn-Alc (Langdon type) allele, while $541 \mathrm{bp}$ fragments, which amplified with primers Vrn-A1-intr_F and Vrn-A1-intr_R1, detect the intact $V R N-A 1$ intron-1.

During investigation of $V R N-A 1$ allelic diversity, it was found that the great majority $(72 \%)$ of $T$. durum varieties with the dominant $V R N-A 1$ carry $7.2 \mathrm{~kb}$ deletion within the $V R N-A 1$ first intron, which characterises the Vrn-Alc (Langdon type) allele (GenBank: KX139172), while only $28 \%$ of varieties were found to carry mutations in the promoter region. The five known allelic variants of the $V R N-A 1$ promoter region were identified by $\mathrm{PCR}$ and sequencing (Figs. 1, 2): Vrn-Ala.1 (GenBank: KX139174), Vrn-A1b.1 (GenBank: KX268729), vrn-Alb.3 (GenBank: KU738895), Vrn-A1b.6 (GenBank: KX139169KX139170), and Vrn-A1i (GenBank: KX139167). The Vrn-Ala.1 allele was found exclusively in varieties of $T$. durum from Russia, where it accounted for half of all $V R N$ Al promoter region alleles. Among other $V R N-A 1$ promoter alleles, Vrn-A1b.6 was also quite frequent (20 varieties), while the canonical Vrn-Alb.1 was detected only in four varieties. For the Ukrainian variety of Kharkovskaya 1, which according to passport data has a winter growth habit, the vrn-A1b.3 allele was identified (Fig. 2). The Vrn$A 1 i$ allele, which differs from vrn-Al by an SNP within the A-tract of the VRN-box (Muterko et al. 2016), was shown for Shirvan 3, Shirvan 5, and GK Basa from Azerbaijan and Hungary, respectively (Fig. 2).
Fig. 1 Electrophoresis of the $V R N-A 1, V R N-B 1$, and $P P D-A 1$ promoter region PCR fragments (three PCR reaction mix per a gel lane). Arrows indicate amplicons corresponding to the $V R N 1$ alleles and the Ppd-A1b haplogroup (AI and AII). Some PCR fragments of $V r n-A 1 b .6$ and the amplicon of $V R N-B 1 . m$ were sequenced and deposited in GenBank under accessions: KX139169-KX139170 and KX139168
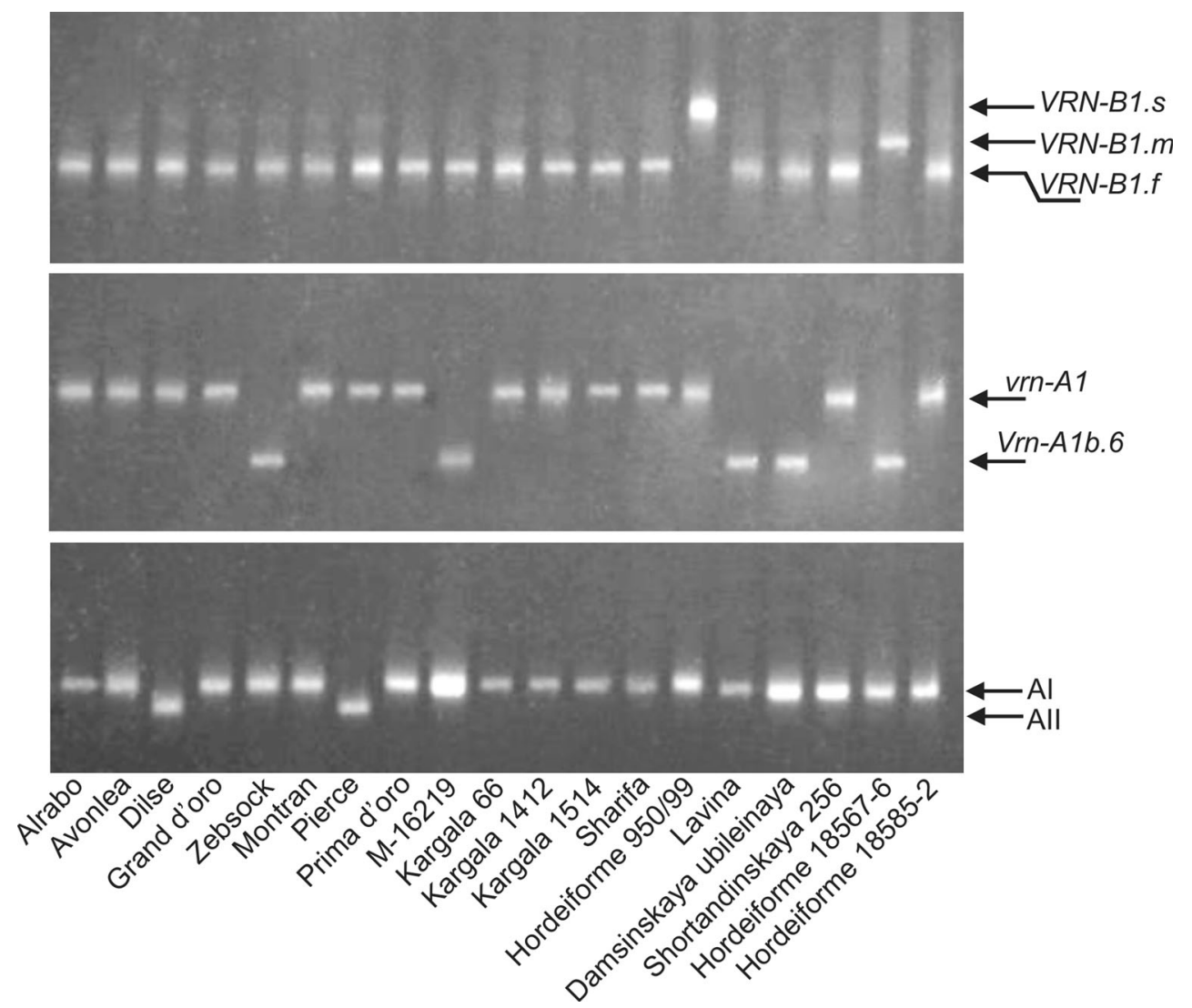
Fig. 2 Allelic variation at the $V R N-A 1$ gene, caused by polymorphism in the promoter region, revealed in varieties of T. durum. a PAGE of the $V R N$ $A 1$ promoter region amplicons, obtained during PCR with primers VRN1AF and VRN1INT1R. b Sequence variation of the VRN-box among different $V R N-A 1$ alleles identified in the present study. Allele name,

VRN-box sequence, and

Genbank accession number are indicated
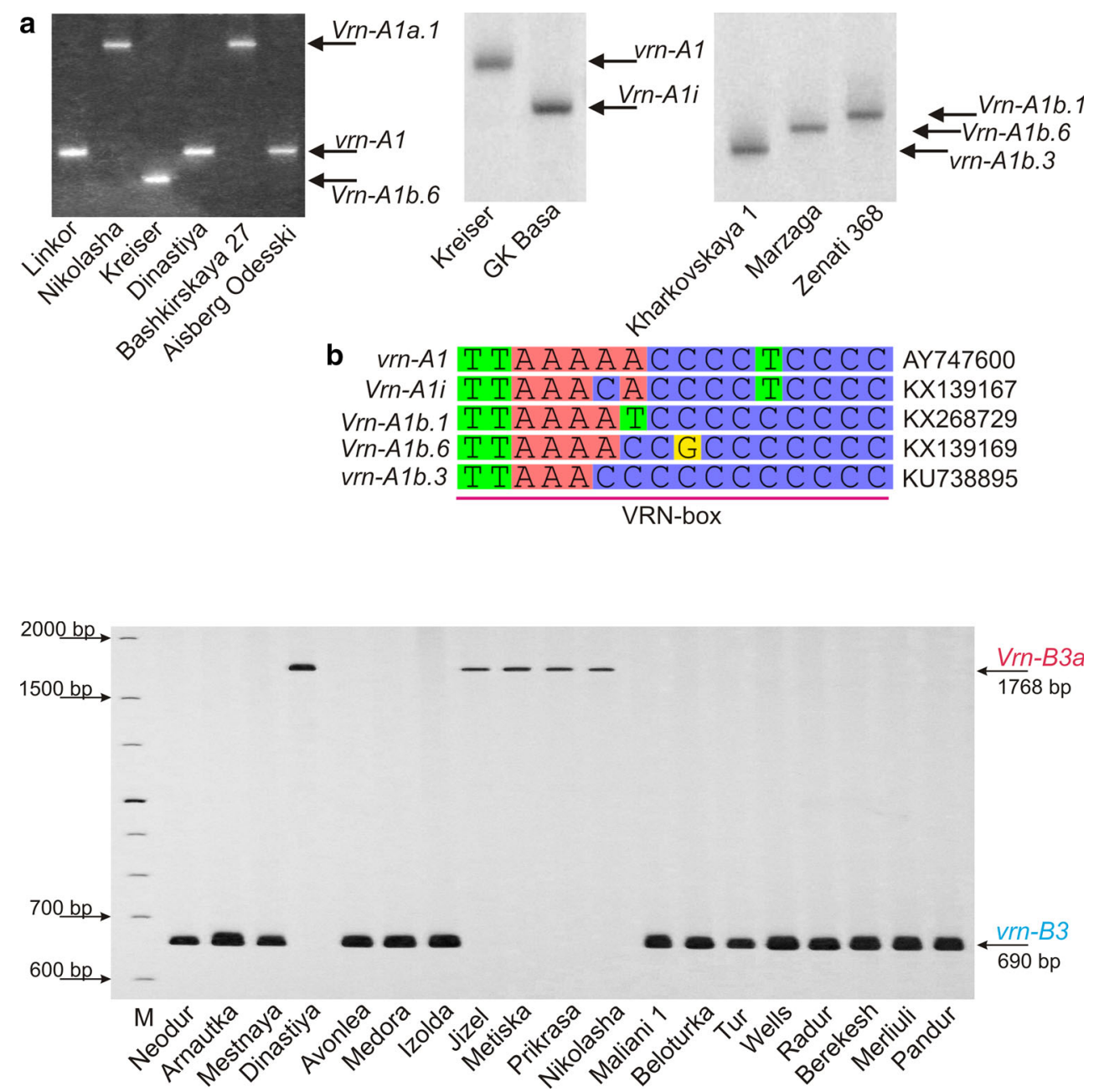

Fig. 3 Electrophoregram of the $V R N-B 3$ promoter region PCR fragments in PAA gel.

Amplicons of the dominant $\mathrm{Vrn}$ $B 3 a$ and recessive $v r n-B 3$ alleles, obtained during multiplex PCR with primers FTB-INS-F, VRN4-B-NOINS-F2, and VRN4-B-NOINS-R, are indicated. Lane M,DNA molecular size marker. A PCR fragment of $V r n-B 3 a$ was sequenced and deposited in GenBank under accession number KX139171
PAGE of the PCR fragments obtained using Pr1 and Pr2 pair primers was performed to distinguish of the $V R N-B I$ promoter sequence variants (Fig. 1). Results demonstrated that 13 varieties of $T$. durum carry the VRN-B1.s allele, containing 7,3 and $2 \mathrm{bp}$ deletions, and the variety Hordeiforme 18567-6 from Kazakhstan has VRN-B1.m with 2 and 3 bp deletions (GenBank: KX139168), while other cultivars are characterized by the intact $V R N-B 1$ promoter ( $V R N-B 1 . f$ allele). Varieties with $V R N-B 1 . s$ predominated in Russia. Investigation of the $V R N-B 1$ first intron by multiplex PCR using primers Ex1/C/F, Intr1/B/ $\mathrm{R} 3$, and Intr1/B/R4 revealed that the dominant alleles of $V R N-B 1$, carrying large deletions within intron-1, were predominant in Ukrainian, Russian, and Kazakhstan varieties (96\%); herewith, for these cultivars, the exceptionally Vrn-B1c (GenBank: KX139173) was identified. This allele also was found in single variety from Turkey (Firat 1) and Canada (Medora). The cultivar of Avonlea from USA alone carries Vrn-Bla. PCR fragments from Hordeiforme 18567-6 migrated through PAA gels slightly slower than amplicons of $v r n-B 1$. However, this difference was not observed under electrophoresis in agarose gels. The difference between these two amplicons is likely to be caused by polymorphism within A-tracts, explaining the change of migration rate in PAA gels.

To distinguish the allelic variants of the $V R N-B 3$ gene, the multiplex PCR using the primers FT-B-INS-F, VRN4B-NOINS-F2, and VRN4-B-NOINS-R was carried out (Fig. 3). In this PCR, fragments migrating at $690 \mathrm{bp}$ indicated the recessive $v r n-B 3$, while $1768 \mathrm{bp}$ fragments detected the dominant $V r n-B 3 a$ allele, containing the $\sim 5.3 \mathrm{~kb}$ retrotransposon insertion in the promoter region. Dominant $V R N-B 3$ alleles are very rare in wheat, so it was surprising that in 28 varieties of $T$. durum, the Vrn-B3a (GenBank: KX139171) was identified (Fig. 3). Among them, the varieties from Ukraine (79 \%) predominated; five varieties were from Russia and only one from Kazakhstan. $V r n-B 3 a$ was revealed only in combination with dominant alleles of VRN-Al (25\%) alone or often with both VRNI genes $(75 \%)$.

The hierarchical cluster analysis results are represented in detail in Fig. 4 and are summarized in Table 2. The 
cladogram from Fig. 4 shows the cluster distribution of varieties of $T$. durum depending on the content of VRN alleles. Additional information about the number of varieties carrying the $V R N$ combination was formed from alleles of previous orders of the given trend, and the percent of varieties carrying this combination relative to all varieties possessing the allele of the current node is indicated for each node. For example, the dominant Vrn-B3a allele is presented in six combinations; herewith, $67.9 \%$ varieties carrying this allele have the combination of $\mathrm{Vrn}$ $A 1 c / V r n-B 1 c / V R N-B 1 . f / V r n-B 3 a$, i.e., characterized by the dominant alleles at the all three $V R N$ genes simultaneously. The nodes of the last order indicate the number of varieties with the given combination of the $V R N$ alleles and their percent in the subset of the T. durum varieties from a given geographical area (country). A total of 22 combinations of VRN alleles were identified. The most common combinations of $V R N$ for varieties of $T$. durum independently of eco-geographical growing area are the $V r n-A 1 c / V R N-B 1 . f /$ vrn-B1/vrn-B3 and Vrn-A1b.6/VRN-B1.f/vrn-B1/vrn-B3 combinations, i.e., predominant varieties containing the dominant $V r n-A l c$ or $V r n-A l b$ allele alone. Three $V R N$ combinations were common for the $T$. durum varieties from Ukraine: (1) all $3 V R N$ are dominant, (2) variety with $v r n-A 1 b .3$, and (3) varieties with winter growth habit (all 3 $V R N$ are recessive). Although four combinations of $V R N$ were specific for varieties from Russia all of them contained the dominant Vrn-Ala allele, which is not found in T. durum from other geographical areas.

Multiplex PCR with primers Ppd-A1proF, durum_Ag5del_F2, and durum_Ag5del_R2 was carried out on varieties of $T$. durum to identify the $P P D-A 1$ allelic variants and haplogroups (Fig. 1). Results showed that the great majority of varieties carry an intact promoter of $P P D$ $A 1$ ( $P p d-A 1 b$ allele) with the AI haplogroup predominant, and the AII haplogroup contains only five varieties. The photoperiod insensitive Ppd-A1a.2 (GenBank: KJ767781) and Ppd-Ala.3 alleles were identified for the Georgian variety Merliuli and variety Metiska from Ukraine, respectively.

\section{Discussion}

Although durum wheat is ranked second in the area sown worldwide after bread wheat (T. aestivum), the varieties of $T$. durum remain poorly studied with regard to the DNA markers of the $V R N$ and $P P D 1$ genes. In the present study, allelic variation at the $V R N-A 1, V R N-B 1, V R N-B 3$, and $P P D-A l$ genes was investigated in a collection of $134 T$. durum varieties, representing 17 countries (Table S1). We compared allelic variation at the $V R N$ genes to determine the specific compositions of these alleles in varieties of $T$. durum originating from different centers of wheat cultivation. During the investigation, the varieties of $T$. durum were analyzed using diagnostic DNA markers for the $V R N$ $A 1, V R N-B 1$, and $V R N-B 3$ promoter regions, and $V R N 1$ intron-1 alleles, and further grouped according to their supposed earliness through sequential clustering in an order, reflecting the influence of $V R N$ genes on flowering time: $V R N-A 1>V R N-B 1>V R N-B 3$. In this clustering, the last order (top tier) is represented by the geographical region (country), where the given combination of the VRN genes was found.

All spring varieties carry a dominant allele of $V R N-A 1$; herewith, varieties with the $V r n-A l c$ allele (containing a large deletion in the first intron) are significantly predominant over accessions carrying mutations within the promoter region of this gene. This is not typical for other tetraploid wheat species, such as Triticum dicoccoides, Triticum dicoccum, Triticum turgidum, or Triticum carthlicum, although it is consistent with findings for Triticum polonicum (Muterko et al. 2016). Five VRN-Al allelic variants carrying mutations within the promoter region were identified. Among them, the dominant Vrn-A1b.1 and Vrn-A1b.6 alleles, which are widespread in tetraploid wheat species, occurred most frequently, while the Vrn-Ali and recessive $v r n-A 1 b .3$ alleles that were frequently recorded in T. turgidum (Muterko et al. 2016), in the present study, were identified in just three and one varieties of T. durum, respectively.

The dominant Vrn-Ala allele, containing an MITE insertion within the promoter region, is more common in spring cultivars of hexaploid wheat $T$. aestivum and became widespread in cultivars after 1970. For this reason, it was supposed that Vrn-Ala is characteristic for hexaploid wheat but not for tetraploid wheat (Yan et al. 2004a). In our previous study, several variants of this allele were found in the A-genome of polyploid wheat. While Vrn-Ala.2 allele was represented only in hexaploid wheat, the Vrn-Ala.l allele was identified also in tetraploid wheat of $T$. dicoccum (Muterko et al. 2016). In the present study, the dominant Vrn-Ala.1 allele was identified in numerous $T$. durum varieties $(20 \%$ of all accessions with a mutant promoter of $V R N-A 1)$ from Russia but was not found in varieties from any others countries. This indicates the specificity of the Vrn-Ala.1 for Russian varieties of T. durum.

In our previous study of the $21 T$. durum accessions from countries outside of Russia, Ukraine, and Kazakhstan, only one accession contained the dominant Vrn-Bla allele (Muterko et al. 2016). Thus, on first glance, it seems that the dominant Vrn-BI alleles are not common in $T$. durum. However, a high frequency of $V r n-B 1 c$ was recorded for varieties from Ukraine, Russia, and Kazakhstan that sharply distinguish them from the $T$. durum varieties from other countries (this study and Muterko et al. 2016). Compared 


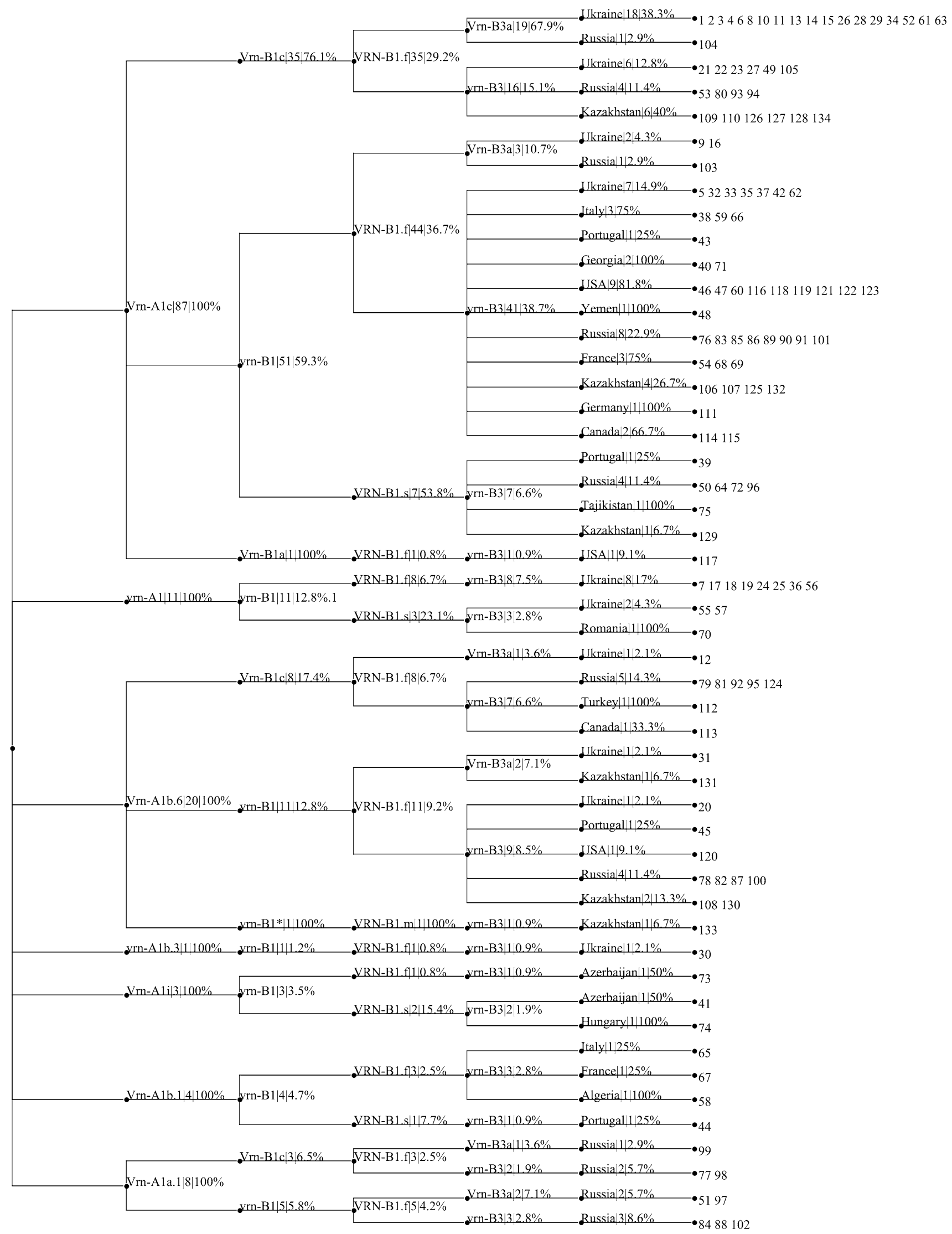


4Fig. 4 Rectangular cladogram, representing the result of controlled sequential hierarchical clustering of the T. durum varieties by $V R N$ genes in an order reflecting their effect on flowering time $(V R N$ $A 1>V R N-B 1>V R N-B 3)$. For each nodes, the $V R N$ allelic variants, number of varieties carrying the combination of the $V R N$ alleles, which was formed from nodes of previous orders of given trend and ratio (percent) of these varieties to subset of varieties containing this node value are separated by "I". Nodes of the last tier indicate index of the T. durum varieties from Table $\mathrm{S} 1$

to other tetraploid wheat species, $V r n-B 1 c$ is frequent in $T$. dicoccoides and present partly in $T$. dicoccum (in contrast to Vrn-Alc) but was not found in T. polonicum, T. turgidum, or T. carthlicum (Muterko et al. 2016). Distribution of $V r n-B 1 c$ among the $T$. durum varieties is similar to that obtained for cultivars of bread wheat (T. aestivum), where $V r n-B 1 c$ was frequent in East Europe and Russia (Milec et al. 2013). This distribution results from previous selections and likely indicates a selective advantage of the Vrn$B 1 c$ allele for these geographical areas.

During the investigation of the $V R N-B 1$ promoter region, we found 13 varieties of $T$. durum carrying the $V R N-B 1$.s allele that has been previously shown to be associated with early flowering of spring wheat accessions (Muterko et al. 2016). Furthermore, the variety of
Hordeiforme 18567-6 from Kazakhstan carries the VRNB1.m allele, which is less frequent in wheat and previously identified only in T. dicoccoides (Muterko et al. 2016).

The Vrn-AIi and VRN-B1.s alleles alone have a relatively weak effect on the vernalization response of wheat (Muterko et al. 2016). The Hungarian variety of GK Basa carrying $V r n-A l i$ and $V R N-B 1 . s$ was grown in the glasshouse under a long photoperiod ( $24 \mathrm{~h}$ day length) and without vernalization. The heading date of this variety was 3 days later than for the accession of hexaploid wheat (PI 186391) with Vrn-Dla allele (Vrn-Dla/VRN-B1.f, facultative growth habit), namely, 145 and 142 days, respectively. This confirms our previous results for the Vrn-Ali/VRN-B1.s genotype (Muterko et al. 2016). However, it should be noted that planting of wheat in this way (under a $24 \mathrm{~h}$ photoperiod) showed corresponding changes in phases of development (steam elongation and flowering time) with an approximate 70 days delay. This later flowering phenotype is a precedent among $T$. durum varieties with a facultative growth habit, and opens new opportunities for breeding of durum wheat cultivars. However, as previously was noted (Muterko et al. 2016), the genetic analysis will be required to confirm the observed association between mutations within the Vrn-

Table 2 Distribution of $V R N$ allelic combinations among varieties of T. durum from different geographical areas

\begin{tabular}{|c|c|c|c|c|c|c|c|}
\hline \multirow[t]{2}{*}{$V R N$ alleles combination } & \multirow[t]{2}{*}{ Number of varieties } & \multicolumn{6}{|c|}{ Frequency $(\%)$} \\
\hline & & America & Europe & Russia & Ukraine & Kazakhstan & Other \\
\hline$V r n-A 1 c / V R N-B 1 . f / V r n-B 1 c / V r n-B 3 a$ & 19 & & & 5 & 95 & & \\
\hline$V r n-A 1 c / V R N-B 1 . f / v r n-B 1 / v r n-B 3$ & 41 & 27 & 18 & 20 & 17 & 10 & 7 \\
\hline$v r n-A 1 / V R N-B 1 . f / v r n-B 1 / v r n-B 3$ & 8 & & & & 100 & & \\
\hline$V r n-A 1 c / V R N-B 1 . f / v r n-B 1 / V r n-B 3 a$ & 3 & & & 33 & 67 & & \\
\hline$V r n-A 1 b .6 / V R N-B 1 . f / V r n-B 1 c / V r n-B 3 a$ & 1 & & & & 100 & & \\
\hline$V r n-A 1 b .6 / V R N-B 1 . f / v r n-B 1 / v r n-B 3$ & 9 & 11 & 11 & 45 & 11 & 22 & \\
\hline$V r n-A 1 c / V R N-B 1 . f / V r n-B 1 c / v r n-B 3$ & 16 & & & 26 & 37 & 37 & \\
\hline vrn-A1b.3/VRN-B1.f/vrn-B1/vrn-B3 & 1 & & & & 100 & & \\
\hline Vrn-A1b.6/VRN-B1.f/vrn-B1/Vrn-B3a & 2 & & & & 50 & 50 & \\
\hline$V r n-A 1 c / V R N-B 1 . s / v r n-B 1 / v r n-B 3$ & 7 & & 14 & 58 & & 14 & 14 \\
\hline Vrn-A1i/VRN-B1.s/vrn-B1/vrn-B3 & 2 & & 50 & & & & 50 \\
\hline Vrn-A1b.1/VRN-B1.s/vrn-B1/vrn-B3 & 1 & & 100 & & & & \\
\hline Vrn-A1a.1/VRN-B1.f/vrn-B1/Vrn-B3a & 2 & & & 100 & & & \\
\hline vrn-A1/VRN-B1.s/vrn-B1/vrn-B3 & 3 & & 33 & & 67 & & \\
\hline$V r n-A 1 b .1 / V R N-B 1 . f / v r n-B 1 / v r n-B 3$ & 3 & & 67 & & & & 33 \\
\hline Vrn-A1i/VRN-B1.f/vrn-B1/vrn-B3 & 1 & & & & & & 100 \\
\hline Vrn-A1a.1/VRN-B1.f/Vrn-B1c/vrn-B3 & 2 & & & 100 & & & \\
\hline Vrn-A1b.6/VRN-B1.f/Vrn-B1c/vrn-B3 & 7 & 14 & & 72 & & & 14 \\
\hline Vrn-A1a.1/VRN-B1.f/vrn-B1/vrn-B3 & 3 & & & 100 & & & \\
\hline Vrn-A1a.1/VRN-B1.f/Vrn-B1c/Vrn-B3a & 1 & & & 100 & & & \\
\hline$V r n-A 1 c / V R N-B 1 . f / V r n-B 1 a / v r n-B 3$ & 1 & 100 & & & & & \\
\hline Vrn-A1b.6/VRN-B1.m/vrn-B1/vrn-B3 & 1 & & & & & 100 & \\
\hline
\end{tabular}


$A l i$ and $V R N-B l$ promoter regions, vernalization response, and flowering time.

Previous studies have revealed no less than four allelic variants of $V R N-B 3$ - two dominant and two recessive (Yan et al. 2006; Chen et al. 2013; Derakhshan et al. 2013). Furthermore, it has been shown that dominant alleles of this gene are less frequent in wheat and in particularly in varieties of T. aestivum (Yan et al. 2006; Zhang et al. 2008; Iqbal et al. 2011; Derakhshan et al. 2013; Chen et al. 2013). In the present study, Vrn-B3a was found in 28 varieties of T. durum. Interestingly, almost all these varieties were from Ukraine. This allows us to assume that currently, the pool of $T$. durum varieties from Ukraine is the largest genetic source of the dominant Vrn-B3a allele in wheat. In addition, our data indicate that in contrast to the currently popular view, the dominant Vrn-B3a allele has an earlier origin in tetraploid wheat, but not hexaploid wheat.

Overall, the 22 allelic combinations at the $V R N$ genes were identified. From them, three were described for varieties from Ukraine and four for Russian. Furthermore, the distinctive features of Ukrainian and Russian varieties include a high frequency of the Vrn-Blc allele and presence of genotypes with both dominant VRN1 genes. The most of Ukrainian varieties carry of the dominant $V R N-A 1$, $V R N-B 1$, and $V R N-B 3$ alleles simultaneously. As is already known, allele frequency is an indicator of the past selection in breeding. For this reason, it is likely that the presence of three dominant $V R N$ alleles in Ukrainian varieties of $T$. durum, leading to the early flowering, is an important regional adaptation, which allows it to avoid the effects of high summer temperatures and phytopathogen infection.

The analysis of the PPD-AI allelic variants herein found that the PI Ppd-A1a alleles are not more frequent in $T$. durum varieties, while for varieties of hexaploid wheat $T$. aestivum from these geographical regions, the photoperiod insensitive alleles (in particularly $P p d-D l a$ ) were permanently introduced during breeding as an agronomically valuable adaptation in the days of the "green revolution". Almost all varieties have the AI haplogroup of Ppd-Alb that is frequent in T. durum (Muterko et al. 2015b). The AII haplogroup of $P p d-A 1 b$ is found only for varieties from USA, Romania, and Russia that is also consistent with the trend of distribution of the PPD-Al haplogroups among different eco-geographic areas (Muterko et al. 2015b). These data indicate that likely none of these haplogroups had been selected in the breeding of varieties of T. durum. In our previous study of 6 tetraploid wheat species, including $T$. dicoccoides, $T$. dicoccum, T. carthlicum, T. turgidum, T. polonicum, and T. durum, the PI Ppd-Ala alleles were not identified, thus reaffirming their later emergence in cultivars of $T$. durum. However, their low frequency, revealed in the present study, indicates a more recent timing of this event, explaining the limited distribution of PI Ppd-Ala alleles among varieties of durum wheat.

\section{Conclusions}

Here, we demonstrate that the American and European varieties of $T$. durum have a similar genotype at the $V R N$ genes, while varieties from Ukraine, Russia, and Kazakhstan are characterized by the specific allelic composition of these genes. In particular, for cultivars from these countries, the most commonly held condition is the presence of dominant alleles for both VRN1 genes simultaneously. Furthermore, Ukrainian varieties provide the largest natural genetic source of the dominant $V r n-B 3 a$ allele, and, hence, among them predominant varieties with carrying dominant alleles of three $V R N$ genes simultaneously $(V R N-A 1, V R N-B 1$, and $V R N-B 3)$. The specificity of Russian varieties can be attributed to the high frequency of the Vrn-Ala.1 allele. In both the cases, the Vrn-B3a and Vrn-Ala.1 alleles were previously identified only in hexaploid wheat, and in the present study, for the first time, the high frequency of these alleles in varieties of tetraploid T. durum wheat was revealed. Similarly, for certain varieties of $T$. durum, the photoperiod insensitive Ppd-Ala alleles were identified for the first time.

Author contribution statement AM carried out the experiments, developed an algorithm of the controlled sequential hierarchical clustering, sequenced of the $V R N$ $A 1, V R N-B 1$, and Vrn-B3a alleles, and wrote the manuscript. RK cloned and sequenced the Vrn-Ali and VrnA1b.6 alleles and contributed to the preparation of the manuscript. EA revised the manuscript, contributed to the discussion, and participated in preparing the manuscript. All authors read and approved the final manuscript.

Acknowledgments We are grateful to Carly Schramm for comments and English polishing of the manuscript. The analysis of $63 \mathrm{~T}$. durum varieties (including all varieties from Russia) was supported by the Russian Science Foundation (Project No. 14-14-00161).

\section{References}

Beales J, Turner A, Griffiths S, Snape JW, Laurie DA (2007) A pseudo-response regulator is misexpressed in the photoperiod insensitive Ppd-Dla mutant of wheat (Triticum aestivum L.). Theor Appl Genet 115(5):721-733

Chen F, Gao M, Zhang J, Zuo A, Shang X, Cui D (2013) Molecular characterization of vernalization and response genes in bread wheat from the Yellow and Huai Valley of China. BMC Plant Biol 13:199

Danyluk J, Kane NA, Breton G, Limin AE, Fowler DB, Sarhan F (2003) TaVRT-1, a putative transcription factor associated with vegetative to reproductive transition in cereals. Plant Physiol 132(4):1849-1860 
Derakhshan B, Mohammadi SA, Moghaddam M, Jalal Kamali MR (2013) Molecular characterization of vernalization genes in Iranian wheat landraces. Crop Breed J 3(1):1-11

Díaz A, Zikhali M, Turner AS, Isaac P, Laurie DA (2012) Copy number variation affecting the Photoperiod-B1 and Vernalization-Al genes is associated with altered flowering time in wheat (Triticum aestivum). PLoS One 7(3):e33234

Distelfeld A, Tranquilli G, Li C, Yan L, Dubcovsky J (2009) Genetic and molecular characterization of the VRN2 loci in tetraploid wheat. Plant Physiol 149(1):245-257

Doyle JJ, Doyle JL (1987) A rapid DNA isolation procedure for small quantities of fresh leaf tissue. Phytochem Bull 19:11-15

Fu D, Szucs P, Yan L, Helguera M, Skinner JS, von Zitzewitz J, Hayes PM, Dubcovsky J (2005) Large deletions within the first intron in $V R N-1$ are associated with spring growth habit in barley and wheat. Mol Genet Genom 273(1):54-65

Huson DH, Richter DC, Rausch C, Dezulian T, Franz M, Rupp R (2007) Dendroscope: an interactive viewer for large phylogenetic trees. BMC Bioinform 8(1):460-466

Iqbal M, Shahzad A, Ahmed I (2011) Allelic variation at the Vrn-Al, Vrn-B1, Vrn-D1, Vrn-B3 and Ppd-D1a loci of Pakistani spring wheat cultivars. Electron J Biotechnol 14(1):1-2

Kippes N, Debernardi JM, Vasquez-Gross HA, Akpinar BA, Budak H, Kato K, Chao S, Akhunov E, Dubcovsky J (2015) Identification of the VERNALIZATION 4 gene reveals the origin of spring growth habit in ancient wheats from South Asia. Proc Natl Acad Sci USA 112(39):E5401-E5410

Milec Z, Sumikova T, Tomkova L, Pankova K (2013) Distribution of different $V r n-B 1$ alleles in hexaploid spring wheat germplasm. Euphytica 192(3):371-378

Muterko AF, Balashova IA, Fayt VI, Sivolap YuM (2015a) Molecular genetic mechanisms of regulation of growth habit in wheat. Cytol Genet 49(1):58-71

Muterko A, Kalendar R, Cockram J, Balashova I (2015b) Discovery, evaluation and distribution of haplotypes and new alleles of the Photoperiod-A1 gene in wheat. Plant Mol Biol 88(1-2):149-164

Muterko A, Kalendar R, Salina E (2016) Novel alleles of the VERNALIZATION1 genes in wheat are associated with modulation of DNA curvature and flexibility in the promoter region. BMC Plant Biol 16(Suppl 1):9

Nishida H, Yoshida T, Kawakami K, Fujita M, Long B, Akashi Y, Laurie DA, Kato K (2013) Structural variation in the $5^{\prime}$ upstream region of photoperiod-insensitive alleles Ppd-Ala and Ppd-B1a identified in hexaploid wheat (Triticum aestivum L.), and their effect on heading time. Mol Breed 31(1):27-37

Shcherban AB, Efremova TT, Salina EA (2012) Identification of a new $V r n-B 1$ allele using two near-isogenic wheat lines with difference in heading time. Mol Breed 29(3):675-685

Takenaka S, Kawahara T (2012) Evolution and dispersal of emmer wheat (Triticum sp.) from novel haplotypes of Ppd-1 (photoperiod response) genes and their surrounding DNA sequences. Theor Appl Genet 125(5):999-1014

Wilhelm EP, Turner AS, Laurie DA (2009) Photoperiod insensitive Ppd-Ala mutations in tetraploid wheat (Triticum durum Desf.). Theor Appl Genet 118(2):285-294

Würschum T, Boeven PHG, Langer SM, Longin CFH, Leiser WL (2015) Multiply to conquer: copy number variations at $P p d-B 1$ and $V r n-A 1$ facilitate global adaptation in wheat. BMC Genet 16:96

Yan L, Loukoianov A, Tranquilli G, Helguera M, Fahima T, Dubcovsky J (2003) Positional cloning of the wheat vernalization gene VRN1. Proc Natl Acad Sci USA 100(10):6263-6268

Yan L, Helguera M, Kato K, Fukuyama S, Sherman J, Dubcovsky J (2004a) Allelic variation at the VRN1 promoter region in polyploid wheat. Theor Appl Genet 109(8):1677-1686

Yan L, Loukoianov A, Blechl A, Tranquilli G, Ramakrishna W, SanMiguel P, Bennetzen J, Echenique V, Dubcovsky J (2004b) The wheat VRN2 gene is a flowering repressor down-regulated by vernalization. Science 303(5664):1640-1644

Yan L, Fu D, Li C, Blechl A, Tranquilli G, Bonafede M (2006) The wheat and barley vernalization gene $V R N 3$ is an orthologue of FT. Proc Natl Acad Sci USA 103(51):19581-19586

Zhang XK, Xiao YG, Zhang Y, Xia XC, Dubcovsky J, He ZH (2008) Allelic variation at the vernalization genes $\mathrm{Vrn}-\mathrm{Al}$, Vrn-B1, Vrn$D 1$, and $V r n-B 3$ in Chinese wheat cultivars and their association with growth habit. Crop Sci 48(2):458-470 